\title{
The Dark Energy Survey: Status and First Science Results
}

\author{
Jelena Aleksić* \\ Institut de Física d'Altes Energies \\ Ed. Cn. Campus UAB, E-08193 Barcelona, Spain \\ E-mail: jelena@ifae.es
}

\section{on behalf of the DES Collaboration}

The Dark Energy Survey (DES) is a next-generation large galaxy survey designed to study the origin of the accelerating Universe and the nature of dark energy. These goals are to be achieved through combination of four distinct probes: baryon acoustic oscillations, abundance and spatial distribution of clusters, weak gravitational lensing, and Type Ia supernovae. The main instrument of the survey is DECam - a state-of-the-art 570 mega-pixel CCD camera, with a large, $\sim 3$ sq. deg. field of view, built by the DES Collaboration and installed at the prime focus of the Victor M. Blanco 4-meter telescope in CTIO, Chile. Over the period of five years, DECam will survey 5000 sq. deg. of the southern sky with unprecedented depth, measuring positions, redshift and shape of almost 300 million galaxies, as well as thousands of clusters and supernovae.

Here are presented the status and some of the first DES science results.

The European Physical Society Conference on High Energy Physics

22-29 July 2015

Vienna, Austria

${ }^{*}$ Speaker. 


\section{The Dark Energy Survey}

The Dark Energy Survey (DES) ${ }^{1}$ is a wide-field survey imaging the $5000 \mathrm{deg}^{2}$ of the Southern sky in five optical to near-infrared filters ( $g r i z Y, 400-1065 \mathrm{~nm}$ ). The principal goal of the survey is to tighten the constraints on the dark energy equation of state (by a factor 3-5 over the previous generation of experiments). This is to be achieved through the combination of four complementary, yet independent probes: galaxy cluster counts, weak gravitational lensing measurements, studies of the large scale structures, and measurements of distances to the Type Ia supernovae [1]. Imaging of $\sim 100$ thousand galaxy clusters of masses greater than $2 \times 10^{14} M_{\odot}$ and shape measurements of $\sim 200$ million galaxies will provide information on the dynamics of the expansion of the Universe as well as the growth of the large scale structures. Further constraints on the expansion will be obtained from clustering measurements of $\sim 300$ million galaxies with redshifts up to $z<1.4$, as well as from brightness measurements of $\sim 3000$ supernovae.

In order to fulfil these objectives the DES Collaboration (composed of $\sim 300$ scientists from U.S., U.K., Spain, Germany, Switzerland and Brasil) has designed, built and commissioned the Dark Energy Camera (DECam)[2]. DECam is a 2.2- $\mathrm{deg}^{2}$ FoV imager mounted at the prime focus of the Victor M. Blanco 4-meter telescope at Cerro Tololo Inter-American Observatory (CTIO), Chile. It is constructed to meet the high-level requirements of the survey, such are excellent image quality, high sensitivity in the near-infrared and low readout noise. The camera itself consists of five element optical corrector, seven filters (ugriz $Y$ and $V R$ ), a shutter with a $60 \mathrm{~cm}$ aperture, and a CCD focal plane. There are 74 CCDs: 62 that are used for science, and 12 that serve for guiding, alignment and focus. The CCDs have 15 microns x 15 microns pixels with a plate scale of 0.263 arcsec per pixel, and the total DECam resolution is $570 \mathrm{Mpx}$. The schematics of the camera and the close-up of the imager are shown in Figure 1.

The survey is to complete its observations in 525 nights, distributed over a period of 5 years ${ }^{2}$. Its observational footprint (Figure 2) is made up of two components: (1) a wide survey, often called the main survey, that will cover $5000 \mathrm{deg}^{2}$ and deliver measurements of hundreds of millions of galaxies, and (2) a supernova discovery survey of $\sim 30 \mathrm{deg}^{2}$ that will provide the light curves for few thousands of Type Ia supernovae. The main survey's observing strategy is designed to optimize the flux calibration, repeating observations in areas up to ten times in each band over the course of the 5-year program (or, in average, 2 survey tilings per filter per year). The final survey depth will be 24 magnitudes in $i$-band. The supernova survey will consist of ten $3 \mathrm{deg}^{2}$-fileds (within the $5000 \mathrm{deg}^{2}$ of the survey), observed in shallow or deep exposures, with many repeated observations, usually separated by one week in time.

\section{Science Verification Period}

Although the "First Light" of DECam took place in early September 2012, the survey itself did not officially start until end of August 2013. The time in between, from November 2012 until February 2013, has been dedicated to the so-called 'Science Verification' (SV) period - a "pilot"

\footnotetext{
${ }^{1}$ http: / / www. darkenergysurvey.org

${ }^{2}$ The DES Collaboration counts on $\sim 30 \%$ of the DECam time per year $(\sim 100$ nights, between mid August to mid February) while the rest of the time the telescope is open to external proposals.
} 

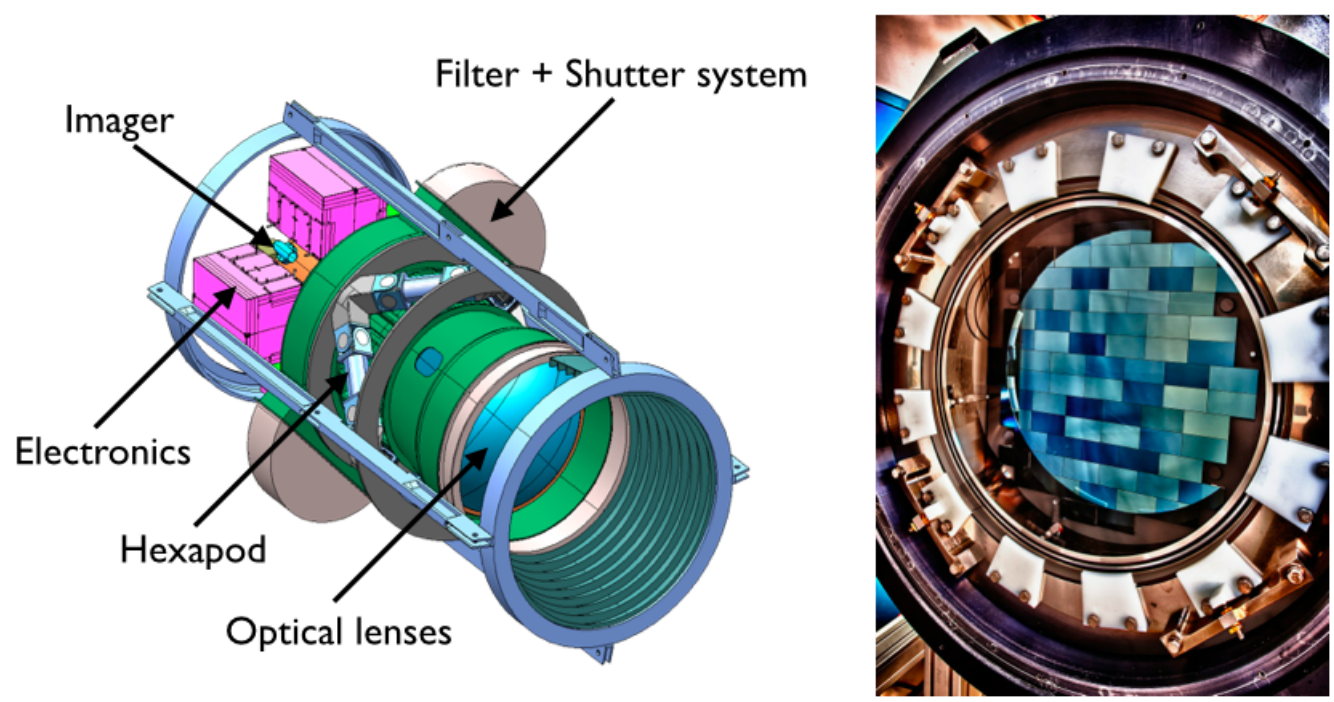

Figure 1: DECam schematics (left) and picture of the imager (right). Credit: DES Collaboration and FermiLab.

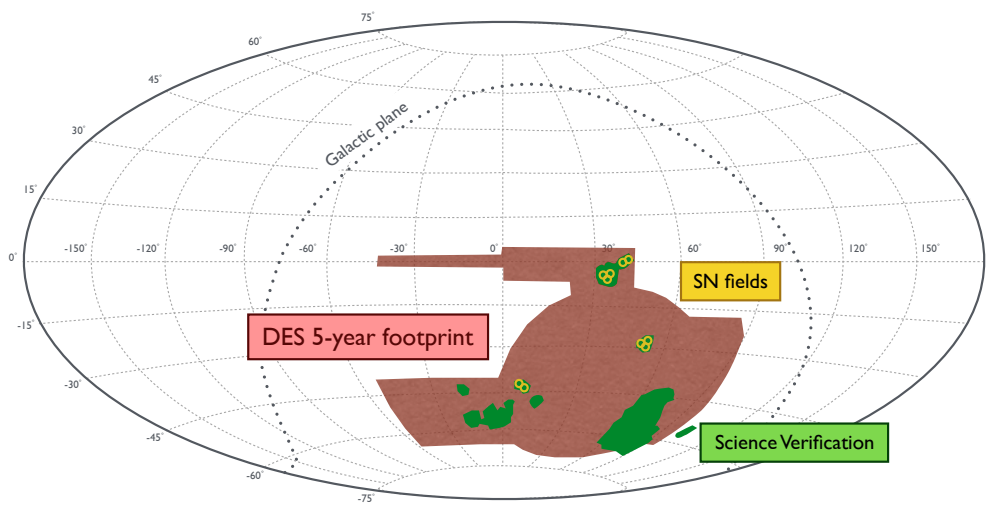

Figure 2: DES 5-year footprint. Dark red color marks the wide survey, the yellow circles are the supernovae survey footprint. The SV fields are pictured in green. Credit: DES Collaboration. 
run devoted to characterisation of the performance of the instruments, as well as to the optimisation and to the fine tuning of the operations, procedures and analysis pipelines.

The SV observations were carried out to the near-to-full survey depth, over several fields covering total of $\sim 250 \mathrm{deg}^{2}$ and selected so that they overlap with the existing measurements from other experiments, as well as to cover some well-known galaxy clusters and supernovae fileds. The SV footprint is marked on Figure 2; the largest continuous area, SPT-E, is approximately $150 \mathrm{deg}^{2}$ in size ( $\sim 3 \%$ of the final DES footprint).

The excellent DECam performance and the good quality of data taken during the SV period have allowed not only for positive evaluation of the specifications and goals proposed before the commissioning, but also for production of first scientific results. In fact, by the time of writing of this Proceeding, $\sim 30$ publications have been produced using the SV data: $\sim 2 / 3$ on science results, and $\sim 1 / 3$ on technical aspects and analysis methods.

The full list of DES publications is available online; the following Section reviews just a few of the first weak lensing results obtained using the SV data.

\section{First Weak Lensing Measurements}

As light from distant galaxies passes through the foreground mass distribution in the Universe, its trajectory gets perturbed, causing distortion and magnification of apparent galaxy shapes. This effect is known as gravitational lensing; it is primarily sensitive to the amount of matter of the lensing body and, in accordance to its effects, distinction is made between the strong, weak and microlensing.

The weak lensing regime has proven to be particularly useful in probing the expansion history and the growth of the structures in the Universe. Shape distorsions caused by this lensing - the cosmic shear - are one of the most statistically powerful cosmological tools (see, e.g. [3]). Shear measurements offer insight into the amount and clustering of dark matter - shear is a tracer of mass, and responds directly to gravity. Furthermore, unlike other dark energy probes, cosmic shear is almost completely unaffected by the complex hydrodynamics of stars, gas and the environment.

On the other hand, precise shear measurements are notoriously hard: these distorsions are a few percent effect at most, far subdominant to the intrinsic shapes of the galaxies. In addition, these measurements must be carried out in the presence of noise, pixelization and distorsions from atmospheric and instrumental effects, all of which can be significantly larger than the shear itself. Then, there are the intrinsic alignments effects, biases from photometric redshift estimates and uncertainties in predicting the density fluctuations in the matter distribution that provide further challenges to the shear measurements. And in order to be able to constrain the dark energy equation of state down to $1 \%$, the uncertainty on cosmic shear must be below $1 \%$.

To overcome these issues, cosmic shear needs to be measured for millions of galaxies, and the systematic errors need to be controlled at the level of $\sim 3$ orders of magnitude smaller than the shape noise on each measurements.

The DES Collaboration has successfully measured shear in $139 \mathrm{deg}^{2}$ of the SV data (the SPTE region) using two independent shear pipelines, IM3SHAPE and NGMIX, producing catalogs of 2.12 and 3.44 million galaxies (4.2 and 6.9 galaxies per square arcmin), respectively [4]. Both of the catalogs have passed a comprehensive suite of null tests that show that they are accurate 
enough to be used for weak lensing science with these data. More details on the pipelines and shear catalogs can be found in [4].

One of the applications of these shear measurements has been presented in [5] and [6]: by converting shear into convergence, we directly get the information on the integrated mass distribution. This mass map was built from shape measurements of 1.1 million SPT-E galaxies at redshifts between 0.6 and 1.2, acting as the sources in the weak lensing regime. It is the largest contiguous lensing mass map made till date by any galaxy survey, and it only covers $\sim 3 \%$ of the final DES footprint. Good agreement was found between the mass map and the distribution of massive galaxy clusters in the foreground (of redshifts between 0.1 and 0.5 , and identified using an independent red sequence cluster finder). Furthermore, potential candidates for super-cluster and voids are identified from over- and under-densities from the combination of these maps. In addition, there is a $6.8 \sigma$ correlation between the mass map and the weighted map of foreground galaxies (that act as lenses, at redshifts between 0.1 and 0.6). This further confirms that the mass projection in the lensing signal of the background sample is good representation of the foreground mass distribution.

Another great strength of cosmic shear measurements as one of the cosmological tools comes in form of 2-point shear statistics: by cross-correlating shapes of galaxies at different redshifts, we can probe the evolution of large scale structures. More details on the 2-point shear statistics measurements and on the photometric redshift estimates for the SV data can be found in [7] and [8], respectively; here we only summarise the proceeding cosmological constraints.

Using the cosmic shear 2-point measurements over three redshift bins, from the $139 \mathrm{deg}^{2}$ of SV data (SPT-E), and after marginalising over 7 systematics parameters and 3 other cosmological parameters, we constrain the matter density of the Universe, $\Omega_{m}=0.36_{-0.21}^{+0.09}$, and the amplitude of fluctuations, $\sigma_{8}=0.81_{-0.26}^{+0.16}$ [9]. We find that our tightest constraint on the degenerate combination is $\sigma_{8}\left(\Omega_{m} / 0.3\right)^{0.5}=0.81 \pm 0.06$, at $68 \%$ c.l. We examine the robustness of our results to the choice of data vector and systematics assumed, and find them to be stable. About $20 \%$ of our error bar comes from marginalising over shear and photometric redshift calibration uncertainties.

The current state-of-the-art cosmic shear measurements from CFHTLenS [10] are mildly discrepant with the cosmological constraints from Planck CMB data [11]; our results are consistent with both datasets. Our uncertainties are $\sim 30 \%$ larger than those from CFHTLenS when we carry out a comparable analysis of the two datasets, which we attribute largely to the lower number density of our shear catalogue. We investigate constraints on dark energy and find that, with this small fraction of the full survey, the DES SV constraints make negligible impact on the Planck constraints.

In the analysis of future DES data from Year One and beyond we aim to be more sophisticated in several ways. Greater statistical power will allow us to constrain our astrophysical systematics more precisely, and algorithmic improvements will reduce our nuisance parameter priors. Forthcoming DES data will provide much more powerful cosmological tests, such as constraints on neutrino masses, modified gravity, and of course dark energy.

\section{Summary}

Dark Energy Survey is the new powerful instrument for studying the dynamics of the expansion of the Universe and the growth of large scale structures. Furthermore, because of its good 
quality the DES data is proving te be a well of non-cosmological science results as well, such are discovery of new dwarf satellite galaxies candidates[12], discovery of high-redshift quasars[13], studies of the Large Magellanic Cloud[14], etc.

The DES SV period has resulted in not only significant improvements in performance and analysis pipelines, but also in a great number of scientific results. Currently in its 3rd year of observations, and in the process of analysing the Year 1 and Year 2 data, new and exciting results from DES are are coming, and will exceed the SV ones in both quality and quantity.

\section{Acknowledgments}

We are grateful for the extraordinary contributions of our CTIO colleagues and the DECam Construction, Commissioning and Science Verification teams in achieving the excellent instrument and telescope conditions that have made this work possible. The success of this project also relies critically on the expertise and dedication of the DES Data Management group.

Funding for the DES Projects has been provided by the U.S. Department of Energy, the U.S. National Science Foundation, the Ministry of Science and Education of Spain, the Science and Technology Facilities Council of the United Kingdom, the Higher Education Funding Council for England, the National Center for Supercomputing Applications at the University of Illinois at Urbana-Champaign, the Kavli Institute of Cosmological Physics at the University of Chicago, the Center for Cosmology and Astro-Particle Physics at The Ohio State University, the Mitchell Institute for Fundamental Physics and Astronomy at Texas A\&M University, Financiadora de Estudos e Projetos, Fundação Carlos Chagas Filho de Amparo à Pesquisa do Estado do Rio de Janeiro, Conselho Nacional de Desenvolvimento Científico e Tecnológico and the Ministério da Ciência, Tecnologia e Inovação, the Deutsche Forschungsgemeinschaft and the Collaborating Institutions in the Dark Energy Survey. The DES data management system is supported by the National Science Foundation under Grant Number AST-1138766.

The Collaborating Institutions are Argonne National Laboratory, the University of California at Santa Cruz, the University of Cambridge, Centro de Investigaciones Enérgeticas, Medioambientales y Tecnológicas-Madrid, the University of Chicago, University College London, the DESBrazil Consortium, the University of Edinburgh, the Eidgenössische Technische Hochschule (ETH) Zürich, Fermi National Accelerator Laboratory, the University of Illinois at Urbana-Champaign, the Institut de Ciències de l'Espai (IEEC/CSIC), the Institut de Física d'Altes Energies, Lawrence Berkeley National Laboratory, the Ludwig-Maximilians Universität München and the associated Excellence Cluster Universe, the University of Michigan, the National Optical Astronomy Observatory, the University of Nottingham, The Ohio State University, the University of Pennsylvania, the University of Portsmouth, SLAC National Accelerator Laboratory, Stanford University, the University of Sussex, and Texas A\&M University.

The DES participants from Spanish institutions are partially supported by MINECO under grants AYA2012-39559, ESP2013-48274, FPA2013-47986, and Centro de Excelencia Severo Ochoa SEV-2012-0234. Research leading to these results has received funding from the European Research Council under the European Union's Seventh Framework Programme (FP7/2007-2013) including ERC grant agreements 240672, 291329, and 306478. 


\section{References}

[1] The DES Collaboration, The Dark Energy Survey, [arXiv:astro-ph/051034 6].

[2] B. Flaugher er al., The Dark Energy Camera, [arXiv: 1504.02900 ].

[3] J. Peacock and P. Schneider,The ESO-ESA Working Group on Fundamental Cosmology, The Messenger 125 (2006) 48.

[4] M. Jarvis et al., The DES Science Verification Weak Lensing Shear Catalogs, [arXiv:1507.05603].

[5] V. Vikram et al., Wide-field lensing mass maps from Dark Energy Survey science verification data: Methodology and detailed analysis, Phys. Rev. D 92 (2015) 022006, [arXiv: 1505. 030 02]

[6] C. Chang et al., Wide-Field Lensing Mass Maps from DES Science Verification Data, Phys. Rev. Lett. 115 (2015) 051301, [arXiv: 1505 .01871].

[7] M. R. Becker et al., Cosmic Shear Measurements with DES Science Verification Data, [arXiv:1507.05598].

[8] C. Bonnett et al. Redshift distributions of galaxies in the DES Science Verification shear catalogue and implications for weak lensing, [arXiv:1507.05909].

[9] The DES Collaboration, Cosmology from Cosmic Shear with DES Science Verification Data, [arXiv: 1507.05552$]$.

[10] C. Heymans et al., CFHTLenS tomographic weak lensing cosmological parameter constraints: Mitigating the impact of intrinsic galaxy alignments, MNRAS 432 (2013) 2433-2453, [arXiv:arXiv:1303.1808].

[11] The Planck Collaboration, Planck 2015 results. XIII. Cosmological parameters, [arXiv:1502.01589].

[12] K. Bechtol et al., Eight New Milky Way Companions Discovered in First-year Dark Energy Survey Data, ApJ 807 (2015) 16 [arXiv: 1503.02584 ].

[13] S. L. Reed et al., DES J0454-4448: Discovery of the First Luminous z âL'ě6 Quasar from the Dark Energy Survey, [arXiv: 1504.03264$]$.

[14] E. Balbinot et al., The LMC geometry and outer stellar populations from early DES data, MNRAS 449 (2015) 1129 [1502.05050]. 